\section{Prognostic performance of Controlling Nutritional Status score in patients with ST segment elevation myocardial infarction treated with primary percutaneous coronary intervention}

\begin{abstract}
Objective: The effect of malnutrition in patients with ST segment elevation myocardial infarction (STEMI) is not fully understood. In this study, we tried to investigate the prognostic consequence of the Controlling Nutritional Status (CONUT) score in patients with STEMI.
\end{abstract}

Methods: In this study, we evaluated the CONUT scores of 1,028 patients with STEMI and examined its relationship with major adverse cardiovascular events (MACE) (all-cause mortality, myocardial reinfarction, and vessel revascularization) during a period of $19.9 \pm 10.3$ months. Patients with CONUT score $\geq 5$ were defined as severely malnourished. Predictors of MACE were assessed by Cox regression analysis, and $p<0.05$ was considered to indicate statistical significance.

Results: MACE was observed in a total of $147(14.3 \%)$ patients. MACE was more frequent in the group with a higher CONUT score $(33.3 \%$ vs. $10.9 \%, p<0.001)$. CONUT score $\geq 5$ was an independent predictor of MACE in the Cox regression analysis (hazard ratio $=2.50,95 \%$ confidence interval: 1.61-3.90, p<0.001). Low ejection fraction, Killip class $\geq 3$ at presentation, thrombolysis in myocardial infarction flow grade $<3$ after intervention, left main artery involvement, and low hemoglobin levels were other independent predictors of MACE in the long-term follow-up. Kaplan-Meier curves showed decreased MACE free survival rates in the high CONUT score group at a mean $19.9 \pm 10.3$ months' follow-up duration (log-rank $p<0.01$ ).

Conclusion: Malnutrition was strongly associated with poor outcomes in patients with STEMI treated using primary percutaneous coronary intervention.

Keywords: malnutrition, Controlling Nutritional Status score, STEMI

\section{INTRODUCTION}

Malnutrition is an important prognostic factor in a wide range of chronic diseases, including malignancy, ischemic stroke, or chronic renal failure (1-3). Several scores have been developed to estimate the degree of malnutrition in these patients. Controlling Nutritional Status (CONUT) score is an integrated approach of both immune system and lipid/protein metabolism. The score was first described by lgnacio de Ulíbarri et al. (4) and was calculated from the serum albumin level, total cholesterol level, and peripheral lymphocyte counts. The utility of the score was mainly demonstrated in various malignancies $(2,5)$. In addition, recent evidence has revealed a poor prognosis in malnourished patients as assessed by the CONUT score, both in acute and chronic heart failure $(6,7)$.

Mortality and morbidity are still high after ST segment elevation myocardial infarction (STEMI) despite advances in treatment options (8). Nutritional management is an essential part of the nonpharmacologic treatment approaches in patients with STEMI. Unfortunately, the vast majority of interventions have focused on patients who are overweight or obese. Latest data indicate that the prevalence of malnourished patients is also common in coronary artery disease (9). However, few studies have addressed the prognostic effect of malnutrition in patients with STEMI (10). Identifying patients

ORIGINAL INVESTIGATION

\author{
Ahmet Zengin iD \\ Mehmet Baran Karataş (iD \\ Yiğit Çanga (D) \\ Gündüz Durmuș (D) \\ Özge Güzelburç (iD \\ Furkan Durak (iD) \\ Ayșe Emre iD \\ Department of Cardiology, University of \\ Health Scienses, Dr. Siyami Ersek Training \\ and Research Hospital; istanbul-Turkey \\ Corresponding Author: \\ Ahmet Zengin \\ $\triangle$ ahmetzengin85@gmail.com
}

Accepted: June 21, 2021

Available Online Date: December 23, 2021

Cite this article as: Zengin $A$, Karataș $M B$, Çanga Y, Durmuș G, GüzelburçÖ, Durak $F$, et al. Prognostic performance of Controlling Nutritional Status score in patients with ST segment elevation myocardial infarction treated with primary percutaneous coronary intervention. Anatol J Cardiol 2022; 26: 23-8.

DOI: 10.5152/AnatolJCardiol.2021.190 
at risk for poor prognosis in the future may help to implement therapeutic interventions, change modifiable risk factors, and increase survival rates. In this study, our aim was to evaluate the role of CONUT score for predicting major cardiovascular adverse events (MACE) in patients with STEMl in the long-term follow-up.

\section{METHODS}

Study design and patient population

Patients diagnosed with STEMI and treated using primary percutaneous coronary interventions ( $\mathrm{pPCls}$ ) between the years 2014 and 2017 were enrolled in this study. The study protocol was approved by the institutional review board. Patients with active infection, malignancy, sepsis, chronic inflammatory diseases, advanced liver disease, and nephrotic syndrome and those receiving steroid or statin therapy were excluded from the study. After exclusion of patients who had incomplete data, the remaining 1,028 patients were included. These final 1,028 patients were divided into 2 groups on the basis of their CONUT score (CONUT $<5$ vs. CONUT $\geq 5$ ). CONUT score was calculated according to the admission serum albumin level, total cholesterol level, and lymphocyte count as presented in Table 1. STEMI was defined as typical chest pain lasting $>30$ minutes (within 12 hours of symptom presentation) associated with $0.1 \mathrm{mV}$ or greater ST segment elevation in 2 or more contiguous leads or newly developed left bundle branch block (11). Demographic, clinical characteristics, and laboratory parameters were collected from hospital records, and follow-up data were obtained from the national health registration system or phone calls.

The mean follow-up duration was $19.9 \pm 10.3$ months. The endpoint of the study was the occurrence of MACE, including all-cause mortality, any vessel revascularization, and myocardial reinfarction. Myocardial reinfarction was defined according to the fourth universal definition.

\section{Revascularization procedure and medications}

All the patients underwent invasive evaluation with standard angiographic techniques according to the established guidelines. A loading dose of $600 \mathrm{mg}$ clopidogrel and $300 \mathrm{mg}$ acetylsalicylic acid was administered on admission. Hundred unit per kilogram heparin or $1 \mathrm{mg} / \mathrm{kg}$ low molecular weight heparin was given after the coronary anatomy was identified. The choice of the stent type (bare metal or drug eluting), use of adjunctive therapies such as thrombus aspiration or bailout glycoprotein Ilb/IIla receptor antagonist infusion, or performing pre-post dilatation was left to the discretion of the operator. All patients were on clopidogrel $75 \mathrm{mg}$ and

\section{HIGHLIGHTS}

- Malnutrition is an under-recognized entity despite its frequent prevalence in patients with ST segment elevation myocardial infarction (STEMI).

- Malnutrition assessed by the Controlling Nutritional Status score was an independent predictor of major adverse cardiovascular events in patients with STEMI treated by primary percutaneous coronary intervention.

- Evaluation of nutritional status should be a part of the management of patients with STEMI. acetylsalicylic acid at discharge, whereas other therapies such as $\beta$ blocker, angiotensin converting enzyme inhibitors, or statins were given unless contraindicated.

Laboratory parameters and echocardiography

Venous blood samples were obtained at admission in the emergency department before $\mathrm{pPCl}$. Blood count analyses were performed using the Beckman Coulter Automated CBC Analyzer (Beckman Coulter, Inc., Fullerton, CA, USA). Total cholesterol, triglyceride, and high-density lipoprotein levels were measured enzymatically, and low-density lipoprotein levels were then calculated using Friedewald formula according to these parameters. After $\mathrm{pPCl}$ procedure, all the patients underwent 2-dimensional and Doppler echocardiographic evaluation to determine left ventricular ejection fraction (LVEF) by an experienced operator (Vivid-5, General Electric Company, Milwaukee, WI, USA).

\section{Statistical analysis}

All data were presented as mean \pm standard deviation for variables with normal distribution and on a median (interquartile range) for variables with non-normal distribution. Categorical variables were reported as numbers and percentages. Continuous variables were checked for normal distribution assumption using the Kolmogorov-Smirnov statistics. Categorical variables were tested using Pearson's chi-square test and Fisher's exact test. Differences between the groups were evaluated using the Mann-Whitney $U$ test or the student t-test when appropriate. Univariate and multivariate Cox regression analyses were performed to investigate the independent predictors of MACE. Variables with $p<0.10$ in the univariate regression analyses were included in the multivariate regression analyses. Survival curves were analyzed using Kaplan-Meier estimation and compared using the log-rank test. All the statistical studies were carried out using Statistical Package for Social Sciences software version 22.0 for Windows (SPSS Inc., Chicago, IL, USA).

\begin{tabular}{lcccc}
\hline Table 1. Calculation of Controlling Nutritional Status score \\
\hline & \multicolumn{4}{c}{ Controlling Nutritional Status score } \\
\cline { 2 - 5 } Variables & Normal & Mild & Moderate & Severe \\
\hline Albumin (mg/dL) & $\geq 3.5$ & $3.0-3.4$ & $2.5-2.9$ & $<2.5$ \\
(score) & $(0)$ & $(2)$ & $(4)$ & $(6)$ \\
Total cholesterol & $\geq 180$ & $140-179$ & $100-139$ & $<100$ \\
(mg/dL) (score) & $(0)$ & $(1)$ & $(2)$ & $(3)$ \\
Lymphocyte & $\geq 1,600$ & $1,200-$ & $800-1,199$ & $<800$ \\
(count/mL) (score) & $(0)$ & $1,599(1)$ & $(2)$ & $(3)$ \\
\hline
\end{tabular}

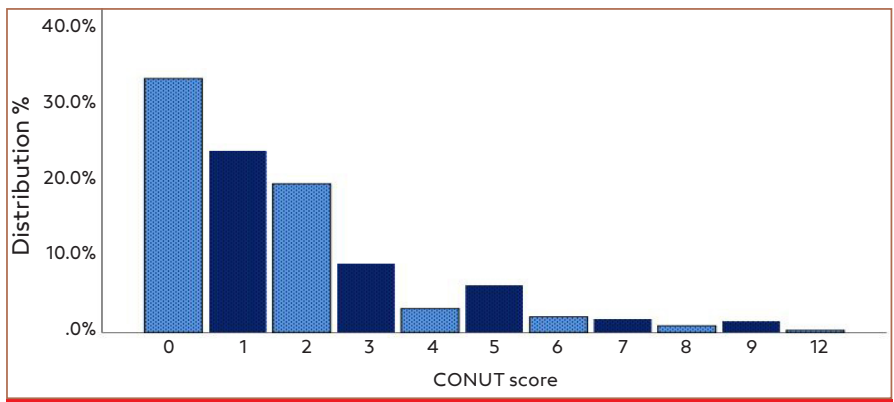

Figure 1. Distribution of the Controlling Nutritional Status score among the study population 


\section{RESULTS}

A total of 1,028 patients with STEMI [mean age $58 \pm 12$ years, $\mathrm{n}=772(74.9 \%)$ men] treated by $\mathrm{pPCl}$ were reviewed. Distribution of CONUT score among the study population is shown in
Figure 1. Patients with a high CONUT score were older $(68 \pm 14$ vs. $56 \pm 12, p<0.001)$, had lower systolic blood pressure $(111.3 \pm 27.8$ vs. $122.2 \pm 20.1, p<0.001)$, and LVEF (37 \pm 16 vs. $44 \pm 11, p<0.001)$. In addition, when compared with the low CONUT score group, patients who were in the high CONUT score group presented

Table 2. Clinical, laboratory, and angiographic characteristics and study outcome parameters

\begin{tabular}{|c|c|c|c|c|}
\hline Variables & Overall $(n=1,028)$ & CONUT $\geq 5(n=129)$ & CONUT <5 (n=899) & $P$-value* \\
\hline \multicolumn{5}{|l|}{ Clinical characteristics } \\
\hline Age, years & $58 \pm 12$ & $68 \pm 14$ & $56 \pm 11$ & $<0.001$ \\
\hline Males, $\mathrm{n}(\%)^{\S}$ & $772(74.9)$ & $102(79.1)$ & $670(74.3)$ & 0.241 \\
\hline Hypertension, n (\%) & $436(42.3)$ & $54(41.9)$ & $382(42.4)$ & 0.908 \\
\hline Diabetes mellitus, n (\%) & $184(17.8)$ & $21(16.3)$ & $163(18.1)$ & 0.619 \\
\hline Previous CAD, n (\%) & $175(17)$ & $18(14)$ & $157(17.4)$ & 0.329 \\
\hline CVA, n (\%) & $23(2.2)$ & $3(2.3)$ & $20(2.2)$ & 0.938 \\
\hline Current smoking, $\mathrm{n}(\%)$ & $666(64.8)$ & 75 (58.1) & $591(65.7)$ & 0.091 \\
\hline Hyperlipidemia, n (\%) & $142(13.8)$ & $12(9.3)$ & $130(14.4)$ & 0.114 \\
\hline Atrial fibrillation, $\mathrm{n}(\%)$ & $34(3.3)$ & $6(4.7)$ & $28(3.1)$ & 0.361 \\
\hline Admission SBP (mm Hg) & $120.8 \pm 21.5$ & $111.3 \pm 27.8$ & $122.2 \pm 20.1$ & $<0.001$ \\
\hline \multicolumn{5}{|l|}{ Angiographic findings } \\
\hline Anterior MI, n (\%) & $567(55)$ & $66(51.1)$ & $501(55.7)$ & 0.701 \\
\hline Left main artery lesion, $n(\%)$ & $34(3.3)$ & $9(7)$ & $25(2.8)$ & 0.012 \\
\hline Multivessel disease, n (\%) & $472(45.8)$ & $69(53.5)$ & $403(44.7)$ & 0.060 \\
\hline Post-procedural flow ${ }^{\S}<$ TIMI 3 n (\%) & $196(19 \%)$ & $43(33.3 \%)$ & $153(17 \%)$ & $<0.001$ \\
\hline Use of stents, n (\%) & $973(94.6)$ & $119(92.2)$ & $854(94.9)$ & 0.761 \\
\hline Gp Ilb/IIla inhibitor use, n (\%) & $411(39.9)$ & 54 (41.9) & $357(39.6)$ & 0.621 \\
\hline \multicolumn{5}{|l|}{ Laboratory analysis } \\
\hline Hemoglobin (g/dL) & $13.8 \pm 1.6$ & $12.5 \pm 1.6$ & $13.9 \pm 1.6$ & $<0.001$ \\
\hline Platelet $\left(10^{3} \mu / L\right)$ & $249 \pm 76$ & $251 \pm 94$ & $249 \pm 73$ & 0.767 \\
\hline HDL cholesterol (mg/dL) & $38 \pm 11$ & $34 \pm 12$ & $38 \pm 12$ & $<0.001$ \\
\hline Triglyceride (mg/dL) & $148 \pm 88$ & $105 \pm 41$ & $154 \pm 91$ & $<0.001$ \\
\hline C-reactive protein $(\mathrm{mg} / \mathrm{dL})$ & $2.3(4.4)$ & $5.1(9.6)$ & $1.9(4.2)$ & $<0.001$ \\
\hline Uric aside (mg/dL) & $5.2 \pm 1.6$ & $5.0 \pm 1.6$ & $5.3 \pm 1.7$ & 0.067 \\
\hline c-Troponin I (ng/mL) & $6.8(44)$ & $8.7(99)$ & $6.7(42)$ & 0.110 \\
\hline \multicolumn{5}{|l|}{ Outcome parameters } \\
\hline MACE, n (\%) & $147(14.3)$ & $49(33.3)$ & $98(10.9)$ & $<0.001$ \\
\hline All-cause mortality, n (\%) & $38(3.7)$ & $29(22.5)$ & $9(1)$ & $<0.001$ \\
\hline Myocardial reinfarction, $\mathrm{n}(\%)$ & $53(5.1)$ & $19(14.7)$ & $34(3.7)$ & $<0.001$ \\
\hline Vessel revascularization, n (\%) & $56(5.4)$ & $6(4.7)$ & $50(5.6)$ & 0.670 \\
\hline
\end{tabular}

*Statistically significant values were given bold.

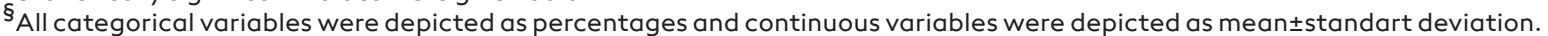

CAD - coronary artery disease; CONUT - Controlling Nutritional Status; CVA - cerebrovascular accident; Gp - glycoprotein; HDL - high-density lipoprotein; LDL - low-density lipoprotein; MACE - major adverse cardiovascular events; MI - myocardial infarction; PAH - pulmonary arterial hypertension; SBP - systolic blood pressure; TIMI - thrombolysis in myocardial infarction 


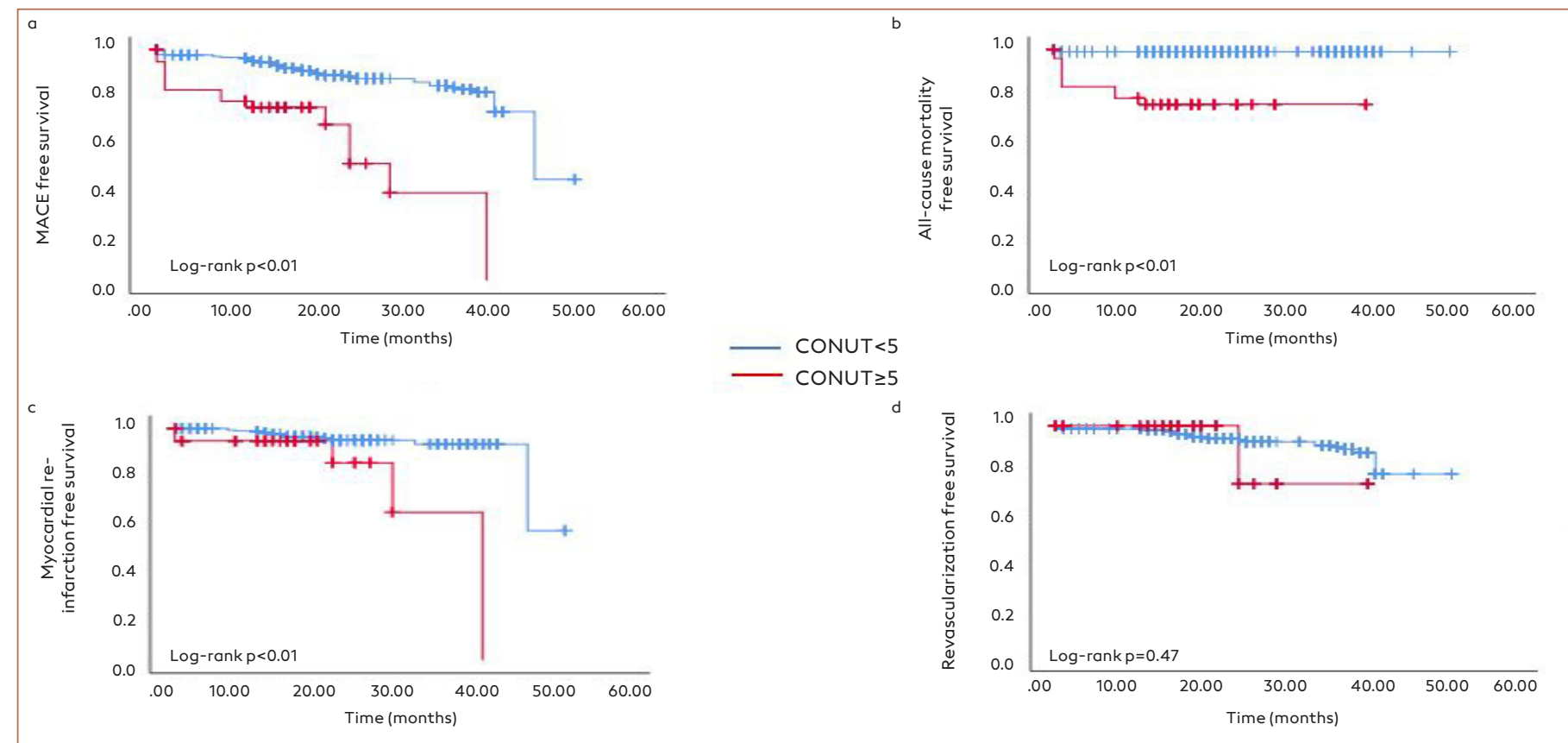

Figure 2. (a) Kaplan-Meier survival curve for major adverse cardiac events, (b) all-cause mortality, (c) myocardial reinfarction, and (d) vessel revascularization according to the Controlling Nutritional Status score

\begin{tabular}{|c|c|c|}
\hline Variables & $\begin{array}{c}\text { Adjusted HR } \\
\text { (95\% CI) }\end{array}$ & $P$-value** \\
\hline Age, years & $1.00(0.98-1.01)$ & 0.756 \\
\hline Left main artery lesion & $13.3(5.02-35.57)$ & $<0.001^{*}$ \\
\hline Ejection fraction $\%$ & $0.98(0.96-0.99)$ & $0.050 *$ \\
\hline Serum creatinine (mg/dL) & $1.36(0.93-1.97)$ & 0.104 \\
\hline Admission heart rate (bpm) & $0.99(0.97-1.00)$ & 0.370 \\
\hline Admission SBP (mm Hg) & $0.99(0.98-1.00)$ & 0.267 \\
\hline Hemoglobin (gr/dL) & $0.84(0.76-0.93)$ & $0.002 *$ \\
\hline CONUT $\geq 5$ & $2.50(1.61-3.90)$ & $<0.001^{*}$ \\
\hline Killip class $\geq 3--4$ & $1.79(1.02-3.10)$ & $0.039 *$ \\
\hline Post-procedural flow < TIMI 3 & $1.65(1.06-2.58)$ & $0.026^{*}$ \\
\hline C-reactive protein (mg/dL) & $1.00(0.99-1.01)$ & 0.425 \\
\hline \multicolumn{3}{|c|}{$\begin{array}{l}\text { *Independent predictors of MACE in the long-term follow-up. } \\
\text { **Statistically significant values were given bold. } \\
\text { CONUT - Controlling Nutritional Status; MACE - major adverse } \\
\text { cardiovascular events; SBP - systolic blood pressure; STEMI - ST } \\
\text { segment elevation myocardial infarction; TIMI - thrombolysis in } \\
\text { myocardial infarction }\end{array}$} \\
\hline
\end{tabular}

more frequently in Killip class $\geq 3-4(23.3 \%$ vs. $7.2 \%, p<0.001)$ and had higher admission heart rates ( $87 \pm 21$ vs. $80 \pm 17, p<0.01)$. Angiographic evaluation demonstrated more frequent left main coronary artery involvement $(7 \%$ vs. $2.8 \%, p=0.012)$ and lower thrombolysis in myocardial infarction (TIMI) flow grade $(33.3 \%$ vs. $17 \%, p<0.001$ ) in the high CONUT score group. Patients in the high CONUT score group had lower serum albumin, total cholesterol, triglyceride, lymphocyte, hemoglobin, and low-density lipoprotein and high-density lipoprotein cholesterol levels. Conversely, they had higher $\mathrm{C}$-reactive protein and serum creatinine levels. More data on the baseline characteristics of study population are detailed in Table 2.
We performed univariate and multivariate Cox regression analyses for all variables to identify the independent predictors of MACE. In univariate regression analyses, age, ejection fraction, admission systolic blood pressure and heart rate, hemoglobin, serum creatinine and C-reactive protein levels, left main coronary artery lesion, CONUT score $\geq 5$, Killip class $\geq 3$, and TIMI flow grade $<3$ after $\mathrm{pPCI}$ were found to be correlated with the occurrence of MACE. In multivariate regression analysis, ejection fraction [hazard ratio $(H R)=0.98,95 \%$ confidence interval $(\mathrm{Cl})$ : 0.96-0.99, $\mathrm{p}=0.050$ ], hemoglobin levels ( $\mathrm{HR}=0.84,95 \% \mathrm{Cl}: 0.76-0.93, \mathrm{p}=0.002)$, presentation with Killip class $\geq 3(\mathrm{HR}=1.79,95 \% \mathrm{Cl}: 1.02-3.10, \mathrm{p}=0.039)$, post$\mathrm{PCI}$ TIMI flow grade $<3(\mathrm{HR}=1.65,95 \% \mathrm{Cl}: 1.06-2.58, \mathrm{p}=0.026)$, CONUT score $\geq 5$ (HR=2.50,95\% Cl: 1.61-3.90, $\mathrm{p}<0.001)$, and left main coronary artery lesion ( $\mathrm{HR}=13.3,95 \% \mathrm{Cl}: 5.02-35.57$, $\mathrm{p}<0.001)$ were independent predictors of MACE in the longterm follow-up (Table 3).

We had also checked tolerance and variance inflation factor (VIF) for all parameters included in the regression model to prevent multicollinearity. According to the multicollinearity statistics, the tolerance values were $>0.1$ and VIF values were $<10$ for all parameters. Therefore, we determined that there was no multicollinearity between each of the variables in the regression model.

MACE was observed in 147 patients (14.3\%), including allcause mortality in 38 patients, myocardial reinfarctions in 53 patients, and vessel revascularizations in 56 patients during the $19.9 \pm 10.3$ months' follow-up period. As shown in Figure 2, Kaplan-Meier curves showed that patients in the high $\mathrm{CO}$ NUT score group had significantly higher rates of MACE, allcause mortality, and myocardial reinfarction with a log-rank of $p<0.01$. However, vessel revascularization was comparable between the groups (log-rank $p=0.47)$. 


\section{DISCUSSION}

In this study, we investigated the association between nutritional status and prognosis in patients with STEMI. Our major finding was that CONUT score $\geq 5$ could predict increased risk of MACE in patients with STEMI in the long-term follow-up.

Nutritional status is a good marker of general condition of patients, including physical, immunologic, and metabolic competence. Scores accepted to estimate the degree of undernutrition may be more efficient than body mass index (BMI) or serum albumin levels alone. The CONUT score uses 3 easily accessible parameters and can be calculated in a simple and cost-effective way (4). In addition, the score is formed from modifiable components, and thus, treatments may be guided according to these parameters in the longterm follow-up period.

One important issue highlighted by this study was the prevalence of undernutrition in patients with STEMI. The ratio of severely malnourished patients as reflected by the CONUT score was $12.5 \%$ in our study. Raposeiras Roubín et al. (12) studied 5,062 patients with acute coronary syndrome and revealed that $11.2 \%$ of the study population had moderate-severe malnutrition according to their CONUT scores (as defined by a CONUT score $\geq 5$ ) similar to our findings. Moreover, up to $20.9 \%$ of the patients who were overweight or obese s were undernourished as assessed by the CONUT score, suggesting that malnutrition was not apparent only in patients with a low BMI. However, neither the patients nor the clinicians are aware of this problem.

Malnutrition was an independent predictor of adverse events in our study. The relation between malnutrition and STEMI and how malnutrition impacts prognosis in the long-term has several explanations. First, undernourished patients frequently represent a more fragile population. Patients with CONUT score $\geq 5$ were older and had lower hemoglobin and higher serum creatinine levels in our study reflecting the underlying general condition and fragility of these patients (13). Second, there is a relationship between inflammation and malnutrition as demonstrated in patients with chronic heart failure (14). Atherosclerosis is a low-grade inflammatory process, and the degree of inflammation is much higher in patients with STEMI (15). Inflammation induces metabolic turnover, muscle catabolism, and decreased serum albumin levels (16). In addition, reduced albumin levels increase blood viscosity, lead to platelet activation, and worsen endothelial functions (17). The adverse effect of low serum albumin and high $\mathrm{C}$-reactive protein levels on long-term prognosis in patients undergoing $\mathrm{PCl}$ has been shown by Wada et al. (18). However, a high degree of malnutrition may be associated with a high atherosclerotic burden owing to increased inflammation, and this can translate into a poor prognosis. The CONUT score also includes peripheral lymphocyte counts and therefore has the advantage and ability to provide information about the immune system, which is closely associated with malnutrition. Circulating cytokines in the acute phase of myocardial infarction suppress lymphocyte counts, and lower levels of lymphocytes are associated with recurrent myocardial infarction in the long-term (19). Third, low ejection fraction or right ventricular infarction induces bowel edema resulting in reduced appetite and food absorption (20). The inverse relationship between total cholesterol levels and CONUT score deserves attention. Patients with low total cholesterol level had higher CONUT score, which had a poor prognosis. One potential explanation is that patients with low total cholesterol levels have more comorbid diseases. The underlying disease lowers cholesterol levels and increases the risk of death. For example, there is a well-known paradox in chronic heart failure, and patients with lower serum cholesterol levels have a poor prognosis (21).

The adverse event rates after STEMI ranged between 4.1\% and $51 \%$ in a recent meta-analysis (22). MACE was seen in 147 patients $(14.7 \%)$ in our study. We identified several predictors of MACE in the long-term follow-up. In agreement with previous results, low LVEF, decreased hemoglobin levels, left main artery involvement, diminished flow after $\mathrm{pPCl}$, and higher Killip class were independent predictors of MACE (23, 24). CONUT score $\geq 5$ was an independent predictor of MACE with a HR of 2.5 in the $19.9 \pm 10.3$ months' follow-up period. When the components of the MACE were detailed, all-cause mortality was more frequent in patients with a higher $\mathrm{CO}$ NUT score. We observed more myocardial reinfarction in the high CONUT score group; however, the ratio of vessel revascularization was comparable between the groups. Patients with a higher CONUT score might have more conservative treatment rather than an invasive approach owing to their general condition as assessed by the attending physician when they are admitted with myocardial reinfarction. This may be an explanation for similar rates of vessel revascularization between groups where myocardial reinfarction was substantially higher.

The CONUT score may identify patients at risk for future adverse events and help develop therapeutic strategies. Malnourished patients may have serum albumin replacement in the acute phase of the myocardial infarction. Nutritional supplements, individualized statin therapy, and referral to a dietician after discharge may be alternative solutions to overcome this problem.

\section{Study limitations}

Our study had a few limitations. The CONUT score was calculated from blood samples obtained before $\mathrm{pPCl}$, and no consecutive calculation was done, which could change the score over time. We were only able to evaluate the all-cause mortality because of missing data. Another limitation was that this was a single center retrospective study with a relatively small sample size.

\section{CONCLUSION}

Malnutrition is a common but under-recognized entity in patients with STEMI. Clinicians should be advised to screen these patients with the CONUT score, and assessing nutritional status should be an integral part of the nonpharmacologic treatment of patients with STEMI. However, future studies are needed to define which scores should be used and whether nutritional support translates into an improved prognosis. 
Conflict of interest: None declared.

Peer-review: Externally peer-reviewed.

Author contributions: Concept - A.Z.; Design - A.Z.; Supervision - Y.C.., A.E.; Fundings - None; Materials - M.B.K., Ö.G.; Data collection \&/or processing - G.D., Ö.G., F.D.; Analysis \&/or interpretation - M.B.K., G.D.; Literature search - A.Z., Y.C.; Writing - A.Z.; Critical review - A.E.

\section{REFERENCES}

1. Almeida HRM, Santos EMC, Dourado K, Mota C, Peixoto R. Malnutrition associated with inflammation in the chronic renal patient on hemodialysis. Rev Assoc Med Bras (1992) 2018; 64: 83744. [Crossref]

2. Suzuki S, Kanaji S, Yamamoto M, Oshikiri T, Nakamura T, Kakeji Y. Controlling Nutritional Status (CONUT) Score Predicts Outcomes of Curative Resection for Gastric Cancer in the Elderly. World J Surg 2019; 43: 1076-84. [Crossref]

3. Naito H, Nezu T, Hosomi N, Aoki S, Kinoshita N, Kuga J, et al. Controlling nutritional status score for predicting 3-mo functional outcome in acute ischemic stroke. Nutrition 2018; 55-56: 1-6. [Crossref]

4. Ignacio de Ulíbarri J, González-Madroño A, de Villar NG, González P, González B, Mancha A, et al. CONUT: a tool for controlling nutritional status. First validation in a hospital population. Nutr Hosp 2005; 20: 38-45.

5. Harimoto N, Yoshizumi T, Sakata K, Nagatsu A, Motomura T, Itoh $\mathrm{S}$, et al. Prognostic Significance of Preoperative Controlling Nutritional Status (CONUT) Score in Patients Undergoing Hepatic Resection for Hepatocellular Carcinoma. World J Surg 2017; 41: 2805-12. [Crossref]

6. Agra Bermejo RM, González Ferreiro R, Varela Román A, Gómez Otero I, Kreidieh $\mathrm{O}$, Conde Sabarís $\mathrm{P}$, et al. Nutritional status is related to heart failure severity and hospital readmissions in acute heart failure. Int J Cardiol 2017; 230: 108-14. [Crossref]

7. Sze S, Pellicori P, Zhang J, Weston J, Clark AL. The impact of malnutrition on short-term morbidity and mortality in ambulatory patients with heart failure. Am J Clin Nutr 2021; 113: 695705. [Crossref]

8. Szummer K, Jernberg T, Wallentin L. From Early Pharmacology to Recent Pharmacology Interventions in Acute Coronary Syndromes: JACC State-of-the-Art Review. J Am Coll Cardiol 2019; 74: 1618-36. [Crossref]

9. Wada H, Dohi T, Miyauchi K, Endo H, Tsuboi S, Ogita M, et al. Combined effect of nutritional status on long-term outcomes in patients with coronary artery disease undergoing percutaneous coronary intervention. Heart Vessels 2018; 33: 1445-52. [Crossref]

10. Deng X, Zhang S, Shen S, Deng L, Shen L, Qian J, et al. Association of Controlling Nutritional Status Score With 2-Year Clinical Outcomes in Patients With ST Elevation Myocardial Infarction Undergoing Primary Percutaneous Coronary Intervention. Heart Lung Circ 2020; 29: 1758-65. [Crossref]

11. Ibanez B, James S, Agewall S, Antunes MJ, Bucciarelli-Ducci C, Bueno H, et al.; ESC Scientific Document Group. 2017 ESC Guidelines for the management of acute myocardial infarction in patients presenting with ST-segment elevation: The Task Force for the management of acute myocardial infarction in patients presenting with ST-segment elevation of the European Society of Cardiology (ESC). Eur Heart J 2018; 39: 119-77. [Crossref]

12. Raposeiras Roubín $S$, Abu Assi E, Cespón Fernandez M, Barreiro Pardal C, Lizancos Castro A, Parada JA, et al. Prevalence and Prognostic Significance of Malnutrition in Patients With Acute Coronary Syndrome. J Am Coll Cardiol 2020; 76: 828-40. [Crossref]

13. Kang $L$, Zhang SY, Zhu WL, Pang HY, Zhang $L$, Zhu $M L$, et al. Is frailty associated with short-term outcomes for elderly patients with acute coronary syndrome? J Geriatr Cardiol 2015; 12: 662-7.

14. Kalantar-Zadeh K, Anker SD, Horwich TB, Fonarow GC. Nutritional and anti-inflammatory interventions in chronic heart failure. Am J Cardiol 2008; 101: 89E-103E. [Crossref]

15. Cusack MR, Marber MS, Lambiase PD, Bucknall CA, Redwood $\mathrm{SR}$. Systemic inflammation in unstable angina is the result of myocardial necrosis. J Am Coll Cardiol 2002; 39: 1917-23. [Crossref]

16. Merker M, Felder M, Gueissaz L, Bolliger R, Tribolet $P$, Kägi-Braun $N$, et al. Association of baseline inflammation with effectiveness of nutritional support among patients with disease-related malnutrition: a secondary analysis of a randomized clinical trial. JAMA Netw Open 2020; 3: e200663. [Crossref]

17. Joles JA, Willekes-Koolschijn N, Koomans HA. Hypoalbuminemia causes high blood viscosity by increasing red cell lysophosphatidylcholine. Kidney Int 1997; 52: 761-70. [Crossref]

18. Wada H, Dohi T, Miyauchi K, Doi S, Naito R, Konishi H, et al. Independent and Combined Effects of Serum Albumin and C-Reactive Protein on Long-Term Outcomes of Patients Undergoing Percutaneous Coronary Intervention. Circ J 2017; 81: 1293-300. [Crossref]

19. Núñez J, Núñez $E$, Bodí $V$, Sanchis J, Mainar L, Miñana $G$, et al. Low lymphocyte count in acute phase of ST-segment elevation myocardial infarction predicts long-term recurrent myocardial infarction. Coron Artery Dis 2010; 21: 1-7. [Crossref]

20. Rahman A, Jafry S, Jeejeebhoy K, Nagpal AD, Pisani B, Agarwala R. Malnutrition and Cachexia in Heart Failure. JPEN J Parenter Enteral Nutr 2016; 40: 475-86. [Crossref]

21. Rauchhaus M, Clark AL, Doehner W, Davos C, Bolger A, Sharma $R$, et al. The relationship between cholesterol and survival in patients with chronic heart failure. J Am Coll Cardiol 2003; 42: 1933-40. [Crossref]

22. Poudel I, Tejpal C, Rashid H, Jahan N. Major Adverse Cardiovascular Events: An Inevitable Outcome of ST-elevation myocardial infarction? A Literature Review. Cureus 2019; 11: e5280. [Crossref]

23. Rasoul S, Ottervanger JP, de Boer MJ, Dambrink JH, Hoorntje JC, Marcel Gosselink AT, et al.; Zwolle Myocardial Infarction Study Group. Predictors of 30-day and 1-year mortality after primary percutaneous coronary intervention for ST-elevation myocardial infarction. Coron Artery Dis 2009; 20: 415-21. [Crossref]

24. Vicent L, Velásquez-Rodríguez J, Valero-Masa MJ, Díez-Delhoyo F, González-Saldivar H, Bruña V, et al. Predictors of high Killip class after ST segment elevation myocardial infarction in the era of primary reperfusion. Int J Cardiol 2017; 248: 46-50. [Crossref] 